\title{
Interference Effects in the Conductance of Multi-Level Quantum Dots
}

\author{
C.A. Büsser, G.B. Martins, K.A. Al-Hassanieh, Adriana Moreo, and Elbio Dagotto ${ }^{1}$ \\ ${ }^{1}$ National High Magnetic Field Lab and Department of Physics, Florida State University, Tallahassee, FL 32306
}

(Dated: November 19, 2018)

\begin{abstract}
Using exact-diagonalization techniques supplemented by a Dyson equation embedding procedure, the transport properties of multilevel quantum dots are investigated in the Kondo regime. The conductance can be decomposed into the contributions of each level. It is shown that these channels can carry a different phase, and destructive interference processes are observed when the phase difference between them is $\pm \pi$. This effect is very different from those observed in bulk metals with magnetic impurities, where the phase differences play no significant role. The effect is also different from other recent studies of interference processes in dots, as discussed in the text. In particular, no external magnetic field is here introduced, and the hopping amplitudes dot-leads for all levels are the same. However, conductance cancellations induced by interactions are still observed. Another interesting effect reported here is the formation of localized states that do not participate in the transport. When one of these states crosses the Fermi level, the electronic occupation of the quantum dot changes, modifying the many-body physics of the system and indirectly affecting the transport properties. Novel discontinuities between two finite conductance values can occur as the gate voltage is varied, as discussed here.
\end{abstract}

PACS numbers:

\section{INTRODUCTION}

Among the several interesting potential applications of nanostructures and nanodevices is their role as small-size test ground for the analysis of the influence of many-body interactions in the physics of strongly correlated electronic systems. These correlation effects are believed to be of crucial important in many bulk materials, such as high-temperature superconductors and manganites, but there is no universal agreement on what formalism is the most appropriate for their study. Verifying the accuracy of simple model Hamiltonians in nanoscale systems can provide an important test of the overall validity of the theoretical formalism. Moreover, several compounds are believed to spontaneously form a complex pattern of nanoislands $\frac{1}{2}$ and, as a consequence, accurate studies of a small number of atoms can produce interesting information even for bulk materials.

In addition, new and novel effects can and do emerge from the quantum behavior of electrons in small environments, which are not obviously present in bulk systems. If quantum dots are considered as parts of electronic circuits, it is important to understand the new properties that may arise due to the discrete nature of the quantized states, the influence of interactions and spin, and the coherence of the wave functions in small systems that requires a fully quantum description of the problem. It is only recently that this complicated physics is starting to be unveiled and novel effects have already been found. A typical case is provided by the Kondo effect ${ }^{2}$, which in the bulk produces an increase in the resistance of a sample below the Kondo temperature $T_{\mathrm{K}}$. On the contrary, in mesoscopic systems such as quantum dots, the same effect actually leads to a decrease in the resistance since the Kondo resonance provides a new channel for transport ${ }^{3.4}$.

The analysis of dots is not a mere academic problem. For example, transport properties at low temperatures across quantums dots (QDs) have received considerable attention in the last few years mainly due the possibility to use these struc- tures in quantum computing and spintronics. More specifically, two coupled quantum dots (2QD) have been proposed to represent tunable qubit circuits ${ }^{5}$, where the spin interaction between the electrons in the dots defines the two states of the qubit. For all these reasons, the study of interacting electrons in nanosystems and its associated quantum transport are very important for progress in condensed matter physics in general.

In several previous studies it has been usually assumed that, e.g., the 2QD have only one localized spin per dot, since only one level is assumed active in each dot. For the success of these proposals, it is important to understand the role played by other levels in the QDs - i.e. considering multilevel dots - providing part of the motivation for the work presented here. In addition, to properly perform these subtle studies it is crucial to carry out the calculations using unbiased techniques that do not assume properties of familiar states that arise from previous experience with bulk materials. Concepts such as Fermi liquids are and must be challenged in this context, since there is no reason to expect that nanoscopic entities will admit a similar description. Ideally, the calculations should make the less possible number of assumptions, and in this framework it is important to develop and use suitable numerical techniques to handle these complicated fully-quantum problems accurately. For this reason, the calculations discussed below were carried out with a powerful numerical method that has been recently developed.

Other interesting investigations in this context have proposed the use of quantum dots as interferometers in the Aharanov-Bohm (AB) geometry with external magnetic fields applied $^{6,7}$. In general, previous efforts have considered a magnetic flux enclosed in two possibles paths for the electrons. In these studies, the tunneling matrix-elements are defined with an explicit phase factor that represents the magnetic flux. These investigations have shown that narrow dips in the conductance could appear as a consequence of phase interference in the $\mathrm{AB}$ circuit, for electrons moving through different pathways. Other investigations have also found conductance can- 
cellations for the case of two quantum dots, individually connected to leads and among themselves ${ }^{8}$. This effect is present even using one-body interactions in the formalism, and it is caused by interference effects between two different paths in the geometry of the problem.

One additional important motivation for the analysis discussed in the present paper is to find alternative sources of interference among wave functions that can also lead to dips in conductance, in the absence of external fields and also in the absence of obviously distinct paths. Effects of this variety were recently addressed in multilevel dots by Kim and Hershfield $^{9}$, where the consequences of having tunneling matrix elements with different signs in a multilevel QD and in an $\mathrm{AB}$ circuit were discussed. These authors showed that the matrix-element signs are important to determine the global phase of the electrons when they cross the QD. Also, arrays of odd-number interacting single-level quantum dots have interesting zeros in the conductance as the gate voltage varies 10 .

In the investigations presented here, we consider a system composed of one or more quantum dots - each with two active levels and including the Hubbard many-body interactions coupled to ideal single-channel leads. In gate-voltage regimes where there is only one electron in the QD, the usual Kondo physics ${ }^{3}$ can be found in the low temperature regime. The coupling between the spin in the QD and the spins in the leads creates a resonance at the Fermi level that contributes to the transport. When a second electron enters in the QD a more interesting physics is observed since now the total spin of the system (singlet or triplet) plays an important role ${ }^{11,12,13}$. One of the main results of our investigations is that the two levels of the dots studied here can act as different channels that individually contribute to the overall conductance with their own phase. This phase plays a role similar to that of a magnetic field in the $\mathrm{AB}$ interferometer, and it can induce constructive or destructive interferences. Below, it is explicitly shown that a phase diference of $\pi$ can exist between the two channels, even if there is no explicit difference between their hopping amplitudes and Coulombic interactions. Only a tiny difference in the energies of the two levels is needed for the effect to develop, as well as a nonzero Hubbard coupling which is required to produce the Kondo physics. It should be remarked that this phase difference is different from the phase messured in some QD experiments 14 since it is internal to the QD. A magnetic field is not necessary to observe this interference.

Others recent investigations have also focussed on systems that present interference processes even without magnetic fields or explicitly different paths. For instance, Hofstetter and Schoeller analyzed lateral quantum dots near the singlet-triplet degeneracy point that occurs from the competition of the Hund rule's coupling, that favors the triplet, and the nonzero energy difference between levels, that favors a singlet state ${ }^{15}$. A dip in the conductance was observed in this region. The study discussed in the present paper includes this singlettriplet transition regime as a special case, but mainly focusses on a different variety of interference effects which are present even for a very small energy difference between the levels in the quantum dots. Our main picture is based on the notion that charge transport can occur with different phases when elec-

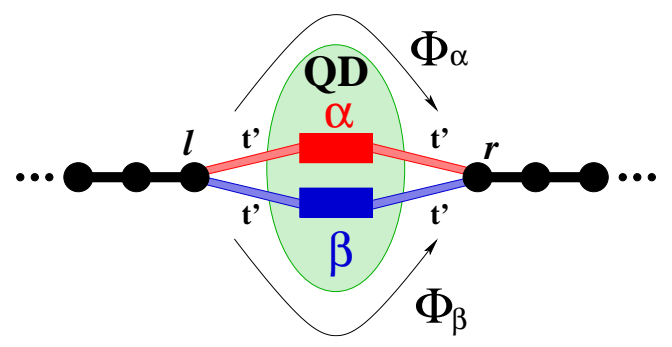

FIG. 1: Schematic representation of the main system studied in this paper, consisting of one quantum-dot with two levels $\alpha$ and $\beta$. The two possible paths carrying different phases $\Phi_{\alpha}$ and $\Phi_{\beta}$ are shown.

trons cross different levels of a quantum dot, rather than a singlet-triplet competition. Alternatively, our results can also be visualized as interference between two Kondo states: one with $S=1$ and the other with $S=1 / 2$.

The organization of the paper is the following: In Sec. IIthe discussion of the formalism, mainly the model and technique, is presented. In Sec. III the case of one dot with two levels is studied in detail, showing the novel interferences in transport. The analysis of the integer-spin Kondo effect, that appears here in the interesting region of parameters, is in Sec. IV] It is well-known that this type of states could enhance the conductance near singlet-triplet transitions ${ }^{11.12}$. A possible intuitive explanation of the effect is given in Sec. $\nabla$ An analysis of other cases where similar effects were found is in Sec. DI and the conclusions are provided in Sec. VII Other effects analyzed in the paper include the possibility of observing localized states near the dots. One such localized state in the QD can cross the Fermi level of the system without contributing to the conductance, but affecting the transport of other levels through the Coulomb interaction. This is shown to lead to discontinuities - as opposed to cancellations - in the conductance vs. gate voltage, a concept that was not discussed before in the literature to our knowledge.

\section{ONE DOT WITH TWO LEVELS: FORMALISM}

The main system that will be analyzed in this paper, and especially in this section, consists of one quantum dot with several interacting levels, connected to ideal leads. It will be argued that - mainly due to the many-body interaction - in a transport process these levels can carry a different phase, as shown schematically in Fig. 1 giving rise to a complex conductance pattern including destructive interferences (i.e. an "internal" Bohm-Aharonov effect appears to be dynamically generated). To model this system, the impurity Anderson Hamiltonian with two levels ( $\alpha$ and $\beta$ ) is used. The portion corresponding to the isolated dot is

$$
\begin{aligned}
H_{\mathrm{dot}}= & \sum_{\lambda=\alpha, \beta \sigma} U / 2 n_{\lambda \sigma} n_{\lambda \bar{\sigma}}+U^{\prime} / 2 \sum_{\sigma \sigma^{\prime}} n_{\alpha \sigma} n_{\beta \sigma^{\prime}}+ \\
& -J \sum_{\sigma \sigma^{\prime}} c_{\alpha \sigma}^{+} c_{\alpha \sigma^{\prime}} c_{\beta \sigma^{\prime}}^{+} c_{\beta \sigma}+
\end{aligned}
$$




$$
\sum_{\sigma}\left[V_{g} n_{\alpha \sigma}+\left(V_{g}+\Delta V\right) n_{\beta \sigma}\right],
$$

where the first term represents the usual Coulomb repulsion between electrons in the same level, which for simplicity is considered equal for both. The second term represents the Coulomb repulsion between electrons in different levels (the $U^{\prime}$ notation is borrowed from standard many-orbital studies in atomic physics problems). The third term represents the Hund coupling $(J>0)$ that favors the alignment of spins, and the last term is simply the energy of the states regulated by the gate voltage $V_{g}$. Note that the level $\alpha$ is assumed to be separated from $\beta$ by $\Delta V$, and by modifying this parameter an interpolation between one- and two-level physics can be obtained. The dot is connected to the leads (represented by semi-infinite chains) by a hopping term with amplitude $t^{\prime}$, while $t$ is the hopping amplitude in the leads. More specifically,

$$
\begin{aligned}
H_{\text {leads }} & =t \sum_{i \sigma}\left[c_{l i \sigma}^{+} c_{l i+1 \sigma}+c_{r i \sigma}^{+} c_{r i+1 \sigma}+\text { h.c. }\right] \\
H_{\mathrm{int}} & =t^{\prime} \sum_{\sigma, \lambda=\alpha, \beta}\left[c_{\lambda \sigma}^{+}\left(c_{l 0 \sigma}+c_{r 0 \sigma}\right)+\text { h.c. }\right]
\end{aligned}
$$

where $c_{l i \sigma}^{+}\left(c_{r i \sigma}^{+}\right)$creates electrons at site $i$ with spin $\sigma$ in the left (right) contact. Site " 0 " is the first site at the left and right of the dot, for each half-chain. The total Hamiltonian is

$$
H_{\mathrm{T}}=H_{\text {dot }}+H_{\text {leads }}+H_{\text {int }} .
$$

Note that for $V_{g}=-U / 2-U^{\prime}+J / 2-\Delta V / 2$, the Hamiltonian is particle-hole symmetric.

Using the Keldysh formalism ${ }^{16}$, the conductance through this system can be written as

$$
\sigma=\frac{e^{2}}{h}\left|t^{2} G_{l r}\left(E_{\mathrm{F}}\right)\right|^{2}\left[\rho\left(E_{\mathrm{F}}\right)\right]^{2},
$$

where $G_{l r}\left(E_{\mathrm{F}}\right)$ is the Green function that corresponds to moving an electron from the left lead to the right one, $E_{\mathrm{F}}$ is the Fermi energy, and $\rho\left(E_{\mathrm{F}}\right)$ is the density of states of the leads (assumed the same left and right, for simplicity):

$$
\rho(\omega)=\frac{-1}{\pi} \operatorname{Im}\left\{\frac{(\omega+i \eta)-\sqrt{(\omega+i \eta)^{2}-4 t^{2}}}{2 t^{2}}\right\} .
$$

To use Eq5 $\left|G_{l r}\right|^{2}$ must be calculated. This quantity has two contributions, one corresponding to transport through path " $\alpha$ " and the other through path " $\beta$ ". From the equations of motion ${ }^{17}$, which correspond to an exact expansion of the Green function $\frac{18}{}$, it can be shown that

$$
G_{l r}=\tilde{g}_{l} t^{\prime} G_{\alpha r}+\tilde{g}_{l} t^{\prime} G_{\beta r},
$$

where $\tilde{g}_{l}$ are the Green function of the first left-contact and $G_{\alpha r}\left(G_{\beta r}\right)$ is the dressed Green functions to move from the state " $\alpha$ " (" $\beta$ ") to the right-contact.

The two terms in Eq 7 involving $G_{\alpha r}$ and $G_{\beta r}$, can be evaluated independently. To calculate the phase difference the following expression was used

$$
i \Delta \Phi=\log \left\{\frac{G_{\alpha r}}{G_{\beta r}} \frac{\left|G_{\beta r}\right|}{\left|G_{\alpha r}\right|}\right\} .
$$

The conductance is proportional to $\left|G_{l r}\right|^{2}$ and, using Eqs. 5] 7 and 8 it can be written as

$$
\sigma=\sigma_{\alpha}+\sigma_{\beta}+\sqrt{\sigma_{\alpha} \sigma_{\beta}} \cos \Delta \Phi,
$$

where $\sigma_{\alpha}$ and $\sigma_{\beta}$ are the partial conductances given by

$$
\begin{aligned}
& \sigma_{\alpha}=\frac{e^{2}}{h}\left[t^{2} \tilde{g}_{l} t^{\prime} \rho\left(E_{\mathrm{F}}\right)\right]^{2}\left|G_{\alpha r}\left(E_{\mathrm{F}}\right)\right|^{2}, \\
& \sigma_{\beta}=\frac{e^{2}}{h}\left[t^{2} \tilde{g}_{l} t^{\prime} \rho\left(E_{\mathrm{F}}\right)\right]^{2}\left|G_{\beta r}\left(E_{\mathrm{F}}\right)\right|^{2} .
\end{aligned}
$$

An exact cancellation of the conductance can occur when $\sigma_{\alpha}=\sigma_{\beta}$ and $\Delta \Phi= \pm \pi$ as a consequence of a destructive interference process. In the same way, the situation with $\Delta \Phi=0$ and $\sigma_{\alpha}=\sigma_{\beta}$ can be considered as a constructive interference.

The zero-temperature Green functions for this problem were calculated using an exact-diagonalization (Lanczos) method $^{19}$ to obtain the ground state and the Green functions of a small cluster containing the many-body interactions. This is followed by an "embedding" procedure to reestablish the rest of the leads ${ }^{20,21}$. The first step in this technique is to separate the system in two portions. One of them is a cluster containing the levels $\alpha$ and $\beta$ (interacting region) and also including the first few sites left and right for each contact. The total number of levels in the cluster is denoted by $L$. For one two-level quantum dot, this corresponds to $L-1$ sites, since one of them has 2 active levels. Since Kondo physics will be important in this analysis, treating exactly not only the dot but also some sites belonging to the leads allows for a proper quantitative consideration of the spin-singlet Kondo "cloud". The other portion in the problem corresponds to the rest of the contacts. Denoting by $\hat{g}$ the exactly calculated Green functions (at $T=0$ ) of the cluster, the rest of the system can be incorporated using the Dyson equation $\hat{G}=\hat{g}+\hat{g} \hat{t} \hat{G}$, where $\hat{t}$ are the matrix elements that connect the cluster with the contacts, and $\hat{G}$ is the dressed Green function.

To take into account possible charge fluctuations inside the cluster, a linear combination of $\hat{g}$ 's calculated with different number of particles is considered. For this purpose, $\hat{g}^{p}=(1-p) \hat{g}_{n}+p \hat{g}_{n+1}$ is defined, where $\hat{g}_{m}$ is the Green function for $m$ particles. With the dressed Green function $\hat{G}^{p}$, the total charge inside the cluster after the embedding is calculated using $Q^{p}=-1 / \pi \int_{-\infty}^{E_{\mathrm{F}}} \sum_{i} \operatorname{Im} G_{i i}^{p}(\omega) d \omega$. On the other hand, the charge in the cluster before the embedding process was $q^{p}=(1-p) n+p(n+1)$. These two quantities must be equal $\left(Q^{p}=q^{p}\right)$, defining a self-consistent equation for $p$.

One of the important scales in this problem is the broadening of levels of the dot due to the interaction with the leads. This energy scale, $\Gamma=\pi t^{\prime 2} \rho\left(E_{\mathrm{F}}\right)^{2}$, defines the Kondo temperature for the case of a spin-1/2 inside the quantum $\operatorname{dot}^{2}$. The coupling values used in this paper are (unless otherwise stated) $U=0.5 t, U^{\prime}=2 / 3 U, J=U-U^{\prime}$, and $t^{\prime}=0.2 t$, that allows for a fast convergence with the cluster size $e^{21}$. Fixing the values for the Coulombic interaction couplings is only for simplicity, to avoid exploring a vast parameter space. Considering $U^{\prime}$ to be smaller than $U$ is reasonable since the latter is expected to be the largest Coulombic interaction. The equation for $J$ is borrowed from atomic physics problems ${ }^{22}$, and 


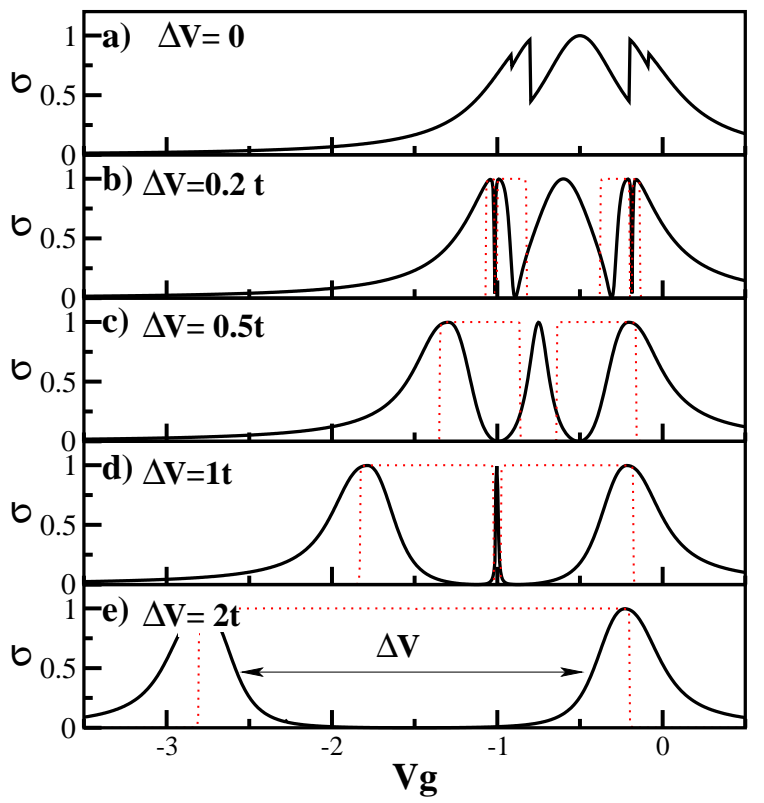

FIG. 2: Conductance for five different values of $\Delta V$. The conductance (in units of $e^{2} / h$ ) is shown in solid lines and the phase difference (divided by $\pi$ ) in dotted lines. When $\Delta V$ is the largest energy ( $\Delta V=2 t$ (e)) two structures similar to those found in a single-level $\mathrm{QD}$, separated by $\Delta V$, appear in the conductance. The case (d) is similar, but since $\Delta V$ is not large enough, an extra thin peak appears around $V_{g}=-1$ (this peak is shown with more detail in Fig. 8. When $\Delta V$ decreases, these structures began to interact giving rise to dips in the conductance, as shown in (b) and (c) for the cases $\Delta V=0.2 t$ and $\Delta V=0.5 t$ ( $\Delta V=0.2 t$ is studied in more detail in Fig. 4. Finally, in the limit $\Delta V=0$, the conductance dips are transformed into discontinuities.

it gives a reasonably small value for the Hund interaction in units of $U$. In addition, we selected the ratio $\Gamma / U$ to be close to the experimental values 23 .

\section{CONDUCTANCE CANCELLATIONS IN MULTILEVEL DOTS}

Using the Lanczos method to evaluate the Green functions of a small cluster followed by the embedding process described in the previous section, we have calculated the conductance, the charge of the QD, and the phase difference Eq 8 as a function of $V_{g}$. Unless otherwise stated, we used the smallest possible cluster size $L$. For the case analyzed in this section this smallest size is $L=4$ that correspond to two "lead" sites and two levels in the dot. In the last part of this section we analyze the convergence of the results increasing $L$. In Fig. 2 we show the conductance for several values of the energy separation $\Delta V$ between the two levels of the dot. At large $\Delta V$ (Fig. 2 (e)) the levels are well separated and the interaction between them is weak. The conductance shows just two wide peaks associated with the standard Kondo effect for each level. Note that the width of these peaks is $U$, and with increasing temperature this broad peak will trans-

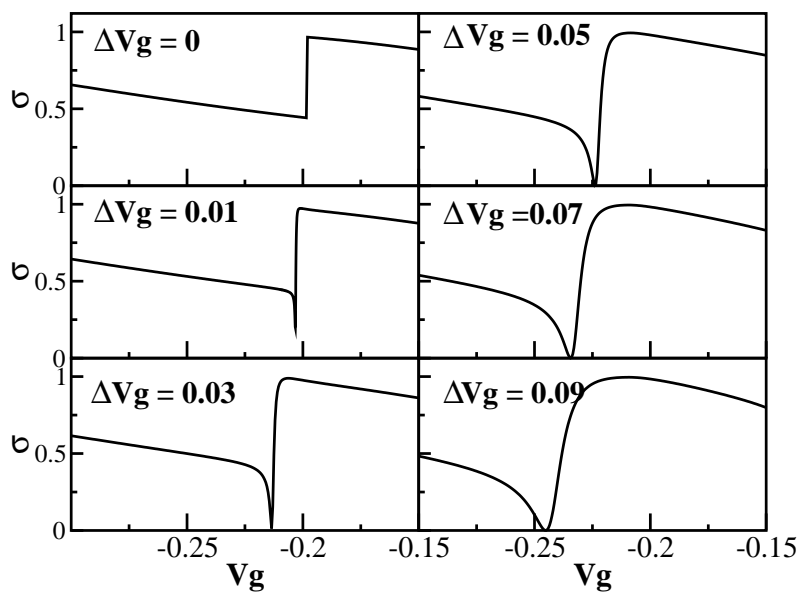

FIG. 3: Conductance (in units of $e^{2} / h$ ) in a narrow range of $V_{g}$ and $\Delta V$. The discontinuity at $\Delta V=0$ is transformed into a dip for finite values of $\Delta V$.

form into two sharper peaks corresponding to the Coulomb blockade. This physics has been discussed at length in previous publications ${ }^{10,13,21}$, and it basically amounts to the behavior of single-level QDs, which is natural at large $\Delta V$.

Far more interesting results are obtained by reducing $\Delta V$. For instance, in Fig. 22(d) a thin central-peak is already observed. This peak seems to be the consequence of a constructive interference between the two possible channels, in a region where a state with total spin $S_{\mathrm{D}}=1$ is formed, as will be discussed more extensively below. Upon further decreasing $\Delta V$ (Figs. 2 (b) and (c) ) the two largest structures began to interact with the thin central peak, which increases its width, and a more complex pattern emerges. With the levels of the dot getting closer it is more likely to have them populated at the same time, and due to the Hund coupling the state with $S_{\mathrm{D}}=1$ has a high probability for the case of two electrons in the dot, giving rise to a spin-1 Kondo effect within the central peak. The other two peaks are associated with $S_{\mathrm{D}}=1 / 2$ and 1 and 3 electrons in the dot. For $\Delta V \leq 0.5 t$, dips in the conductance are clearly observed, and later we will show that these cancellations are related to interference processes. Finally, at $\Delta V=0$ (Fig. 22a)) several discontinuities appear in the conductance. It will be argued that these discontinuities are a consequence of the existence of localized states at the dot. These states are not of direct relevance for the transport of charge, but when the gate voltage moves them below the Fermi level of the leads they simply increase the charge of the quantum dot, which indirectly affects transport through the Coulombic interaction. Note that, for $\Delta V=0$, the phase difference between levels $\alpha$ and $\beta$ is zero, since the Green functions $G_{\alpha 2}$ and $G_{\beta 2}$ are equal. Thus, no destructive interferences are observed, but discontinuities can still occur.

In Fig. 3 it is shown how one of the conductance discontinuities is rapidly transformed into a dip as $\Delta V$ increases from zero. In Figs. 4 5 and 6 we can observe in detail the case with $\Delta V=0.2 t$. Figure 4 shows the conductance together with the phase difference and the partial conductance. Clearly, the zeros of the conductance are in regions where $\Delta \Phi=\pi$. Then, 


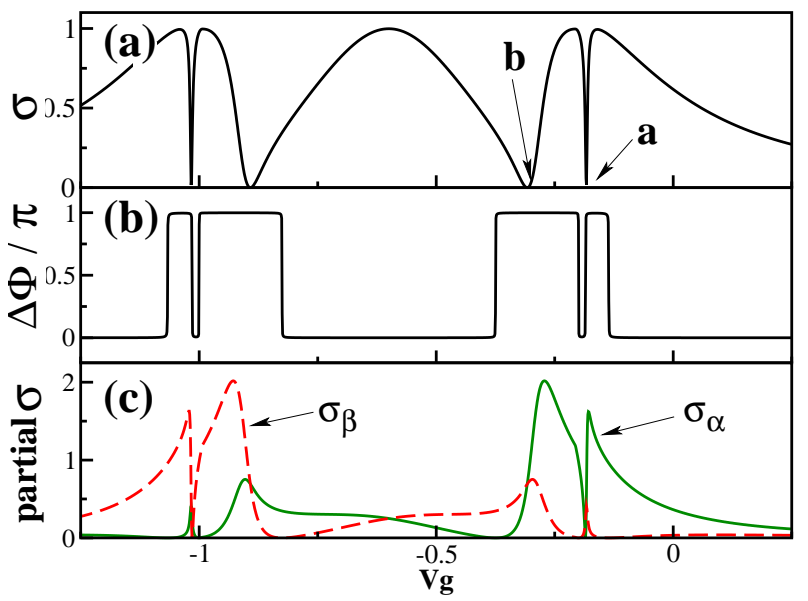

FIG. 4: Conductance (in units of $e^{2} / h$ ) (a), phase difference (divided by $\pi$ ) (b), and partial conductances (c) for $\Delta V=0.2 t$. The dips, indicated by "a" and " $b$ " in (a), are in regions where the phase difference jumps to $\pi$ producing an exact cancellation when $\sigma_{\alpha}=\sigma_{\beta}$.

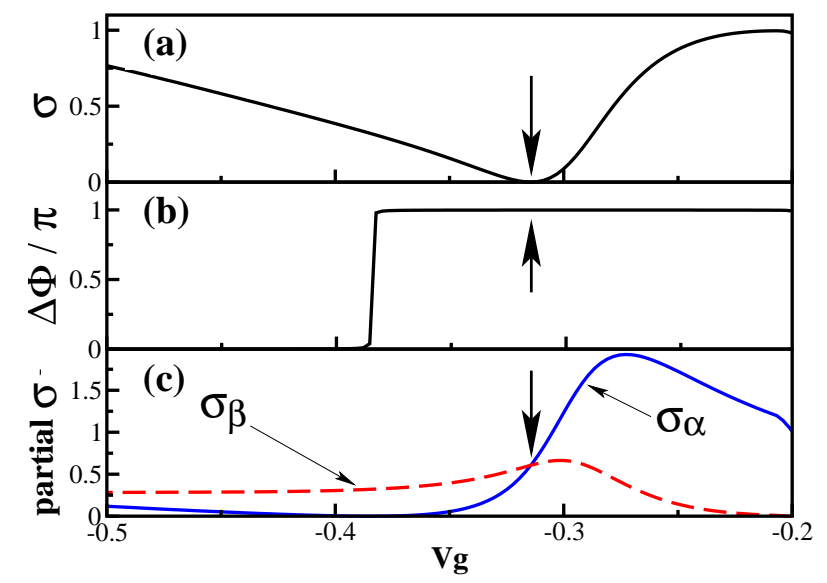

FIG. 5: Conductance (in units of $e^{2} / h$ ), phase difference, and partial conductances for $\Delta V=0.2 t$ for the "b" dip of Fig. 4]a). Thicks arrows shows the gate potential where the conductance cancells, $\Delta \Phi=\pi$ and $\sigma_{\alpha}=\sigma_{\beta}$.

from $\mathrm{Eq} 9$ the dips must occur when the partial conductance $\sigma_{\alpha}$ and $\sigma_{\beta}$ take the same values. More detail for dip "b" is shown in Fig. 5 where thick arrows point to the zero in the conductance and the location where $\sigma_{\alpha}=\sigma_{\beta}$. The dot charge for the same $\Delta V$ is shown in Fig. 6 The central peak in the conductance occurs in the regime where two electrons are in the dot, in different levels. It can also be observed that the total charge at the QD $\left(\sum_{\sigma} n_{\alpha \sigma}+n_{\beta \sigma}\right)$ does not show the characteristic sharp plateauxs of the Coulomb blockade regime: here the electrons are entering in the QD in a smoother way since the Coulomb interaction is comparable to the hopping amplitude $t$ (Fig. 6b) ).

Similar results but for the case of $\Delta V=t$ are shown in Fig. 7 In this situation, the large $\Delta V$ well separates the levels $\alpha$ and $\beta$, and as a consequence one of the levels is charged almost completely before some charge starts to enter into the other level (Fig. 7 ( $\mathrm{c}$ )). The latter occurs at the $V_{g}$ where the

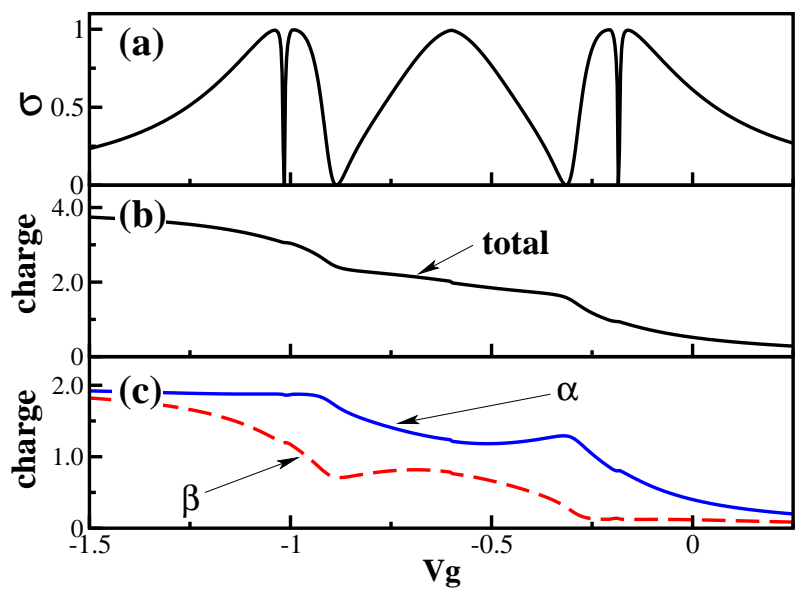

FIG. 6: Conductance (in units of $e^{2} / h$ ), total charge of the QD, and charge at each level for the case $\Delta V=0.2 t$. The charge of the level $\alpha$ is indicated with a continuos lines, while the charge of $\beta$ is shown with a dashed one. The central peak emerges in the regime where there are two electrons in the dot, in different levels.

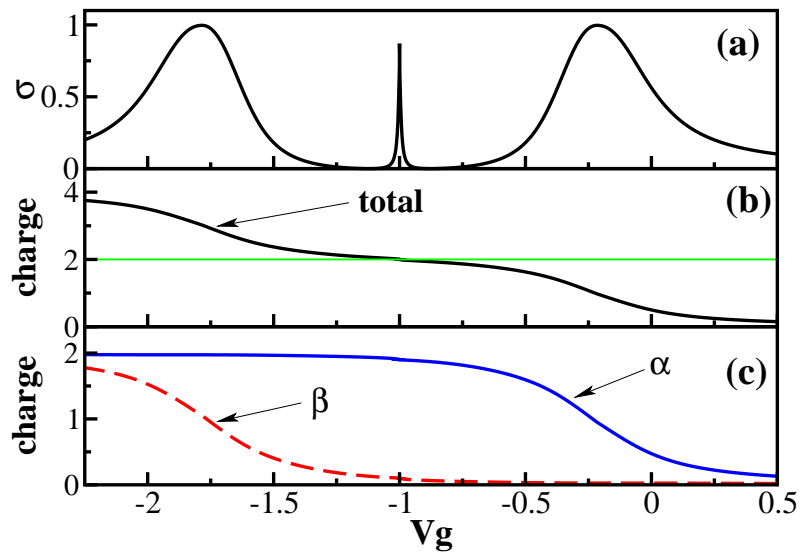

FIG. 7: Conductance (in units of $e^{2} / h$ ) and charges for the case $\Delta V=t$ as a function of the gate potential. These results should be compared against those of Fig. 6

conductance peak is observed.

To better understand the physics associated with the thin central peak for the case $\Delta V=t$ shown in Fig. [2] the conductance and the phase for this case is presented in more detail in a narrow $V_{g}$ range in Fig. 8 We can observe that the partial conductances $\sigma_{\alpha}$ and $\sigma_{\beta}$ are individually small $(<0.25)$ but as the phase between the two channels is 0 they contribute in a constructive way, inducing a peak in the total conductance.

Our results have been obtained solving exactly a relatively small cluster and, then, approximately reaching the bulk limit using the Dyson equation. It is important to verify that our results are robust if the cluster size increases. In Fig. 9 the convergence of the conductance with increasing cluster size at $\Delta V=0.2 t$ is shown. Remarkably, the diference between $L=8$ and $L=20$ is less than $0.5 \%$, showing that the results presented here are nearly exact. The fast convergence is a consequence of the local characteristics of the dot, namely all the impor- 


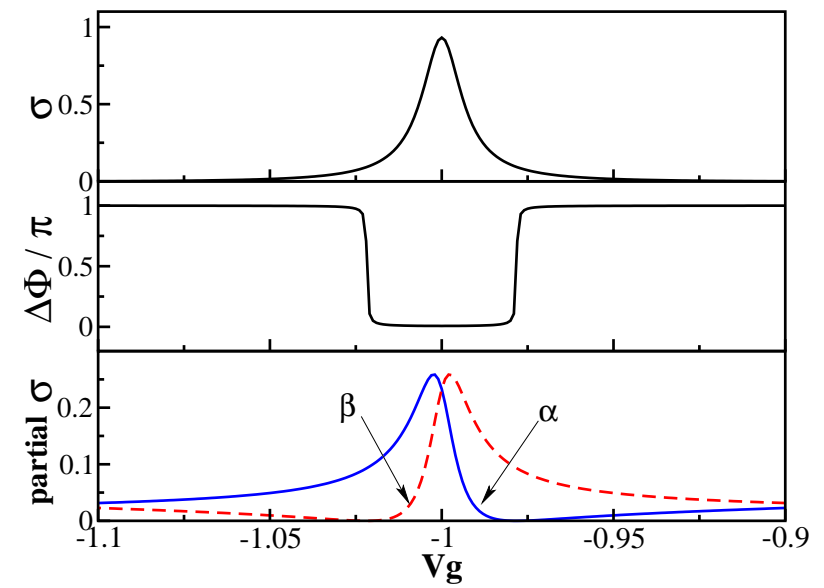

FIG. 8: Conductance (in units of $e^{2} / h$ ), phase difference and partial conductances for $\Delta V=t$ for the central peak shown in Fig. 2]d). The partial contributions to the conductance $\left(\sigma_{\alpha}\right.$ and $\left.\sigma_{\beta}\right)$ have the same shape as in Fig. 4 but now the phase difference between them is zero. This phase difference creates a peak where in the other situation appears a dip.

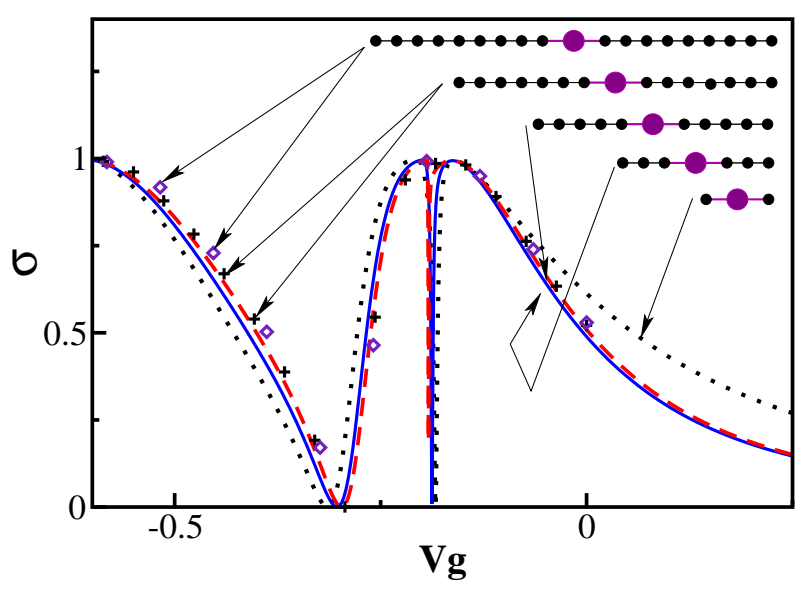

FIG. 9: Convergence of the conductance with the size of the exactlysolved cluster $L$ for the case $\Delta V=0.2 t$. Dotted lines are for $L=4$, continuous thin lines for $L=8$, and dashed lines for $L=12$. Extra points for $L=16$ and $L=20$ are shown with crosses and diamonds, respectively.

tant physics effects are contained in the small cluster solved exactly. The fast size convergence shows that even using the smallest cluster, the results are already qualitative correct.

It is important to remark that the cancellations observed here do not crucially depend on the couplings $U^{\prime}$ and $J$, since they are present in our results even for $U^{\prime}=J=0$, as shown in Fig. 10 Also for completeness, in Fig. 11 the case of a large $U^{\prime}=2 t$ is shown. Even though the situation $U^{\prime}>U$ is unphysical - since the Coulomb repulsion in the same level should be larger than for electrons in different levels - it is interesting to note that the $U^{\prime}$ energy plays a role similar to that of $\Delta V$. Since the energy to have a second electron in $\beta$ is related to $U^{\prime}-J+\Delta V$, for a large $U^{\prime}$ (with a $J$ with the same sign as considered before) the system reacts as with large $\Delta V$. Figure

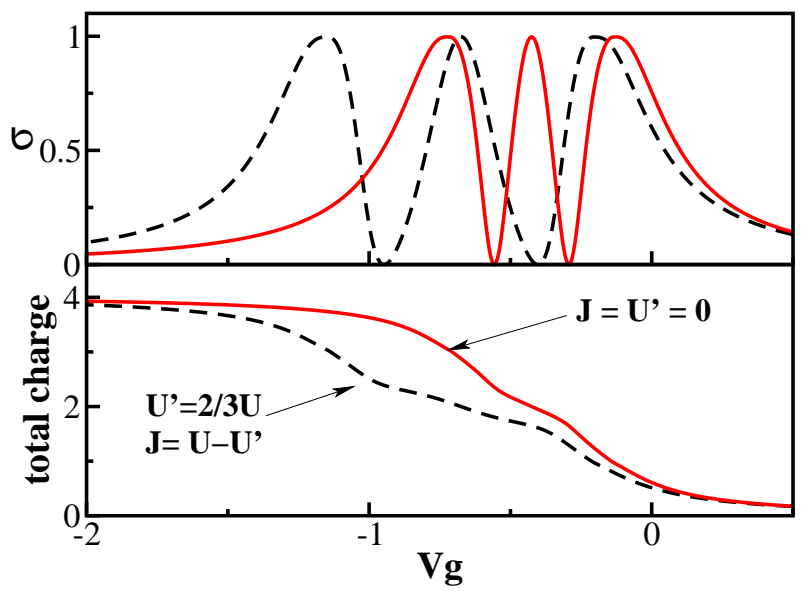

FIG. 10: Study of the conductance and total charge when $U^{\prime}$ and $J$ are not active. Shown are results for the case $U^{\prime}=J=0$ (solid line) compared with the previously shown results at $U^{\prime}=2 / 3 U$ and $J=U-$ $U^{\prime}$, both at $\Delta V=0.35$. The effects are qualitative the same in both cases.

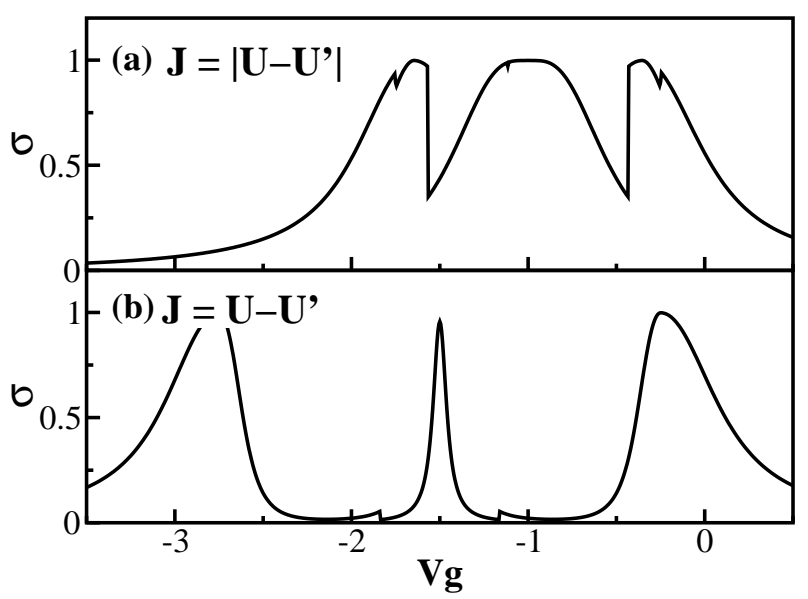

FIG. 11: Conductance (in units of $e^{2} / h$ ) with large $U^{\prime}=2 t$. In (a), it is shown the case with a ferromagnetic Hund coupling $J=\left|U-U^{\prime}\right|$ and in (b) with antiferromagnetic $J=U-U^{\prime}$. Both figures are for $\Delta V=0$.

11 contains two differents cases for $\Delta V=0$, one of them with a ferromagnetic Hund coupling $J=\left|U-U^{\prime}\right|>0$ (a) and the other with an antiferromagnetic coupling $J=U-U^{\prime}<0$ (b). In the first case, since the Hund coupling is proportional to $U^{\prime}$, we observe the three-peaks structures for the conductance with a central-peak associated to the $S_{\mathrm{D}}=1$ states. In the second case, $U^{\prime}$ and $J$ try to eliminate the $S_{\mathrm{D}}=1$ states, and the conductance has a similar structure as shown in the previously study example with $\Delta V=t$ (see Fig. [2]d)).

\section{INTEGER-SPIN KONDO EFFECT}

Recently, transport through a dot connected to two leads has been experimentally studied as a function of the dot level separation $(\Delta V)$, tuned by an external magnetic field. For a 


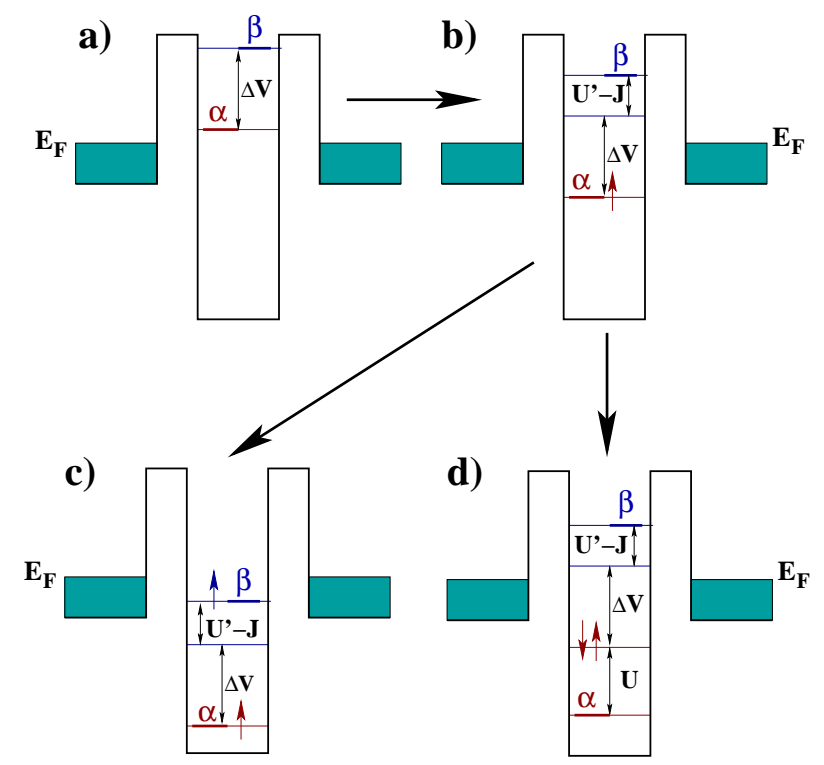

FIG. 12: Schematic representation of the different situations that could occur in a two-level QD as the gate voltage changes. For more details see the text.

dot with an even number of electrons, it has been shown that the Kondo correlations formed between the $S_{\mathrm{D}}=1$ triplet state of the dot and the spins of the leads has a strong influence on the current 11 . This problem was theoretically studied using a mapping onto a two-impurity Kondo mode $1^{12,13}$.

Figure 12 shows schematically the different situations that could arise as the number of electrons inside the QD is changed using the gate voltage. Starting from the upper left (a), we have the case where the gate potential $V_{g}$ is above the Fermi level and there are no electrons inside the QD. Level $\alpha$ is separated from level $\beta$ by the energy $\Delta V$. Decreasing $V_{g}$, as shown in Fig 12 b), one electron populates level $\alpha$, and the energy to add an extra electron in $\beta$ will be $\Delta V+U^{\prime}-J$, since an extra energy $U^{\prime}$ is paid due to the Coulomb repulsion and a $-J$ energy due to the spin coupling. The system now is in the Kondo regime for transport if $T<T_{\mathrm{K}}$. Decreasing even further $V_{g}$, a second electron could enter the dot. The spin of this second electron defines the transport properties since the total spin of the QD can be $S_{\mathrm{D}}=1$ (with a Kondo resonance for temperatures below $\left.T_{\mathrm{K}(\mathrm{S}=1)}\right)$ or $S_{\mathrm{D}}=0$ with no Kondo effect. In the rightlower frame (d) the second electron entered in the lower energy state $\alpha$. The energy of this configuration will be of the form $E_{S=0}=2 V_{g}+U$. On the other hand, if the second electron enters to the dot with the same spin as the first one forming an $S_{\mathrm{D}}=1$ state, we will have the case showed in the left-lower frame (c). The energy of this state in the Kondo regime will be of the form $E_{S=1}=2 V_{g}+\Delta V+U^{\prime}-J-T_{\mathrm{K}(\mathrm{S}=1)^{13}}$. For $\Delta V>U-U^{\prime}+T_{\mathrm{K}(\mathrm{S}=1)}$ we expect a transition from the $S=1$ state (right-lower frame) to the $S=0$ (left-lower frame).

Note that the spin of the dot $S_{\mathrm{D}}$ is not a well-defined quantum number, since the QD is not isolated. In the $L=4$ cluster, we found that the ground state used for the calculations has a spin $S_{\mathrm{D}}=0.7426$ (calculated from the relation

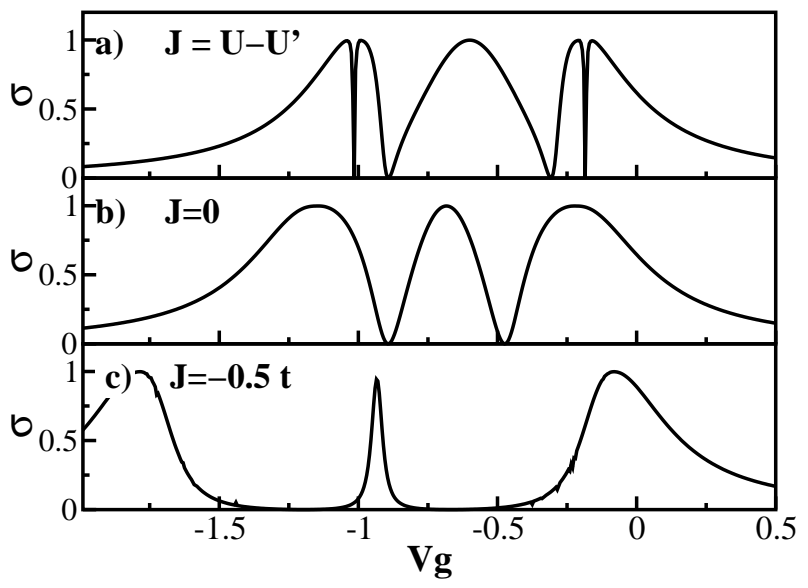

FIG. 13: Conductance (in units of $e^{2} / h$ ) for differents $J$ couplings. (a) corresponds to the case $J=U-U$, (b) is at $J=0$, and (c) is for a large negative case $J=-0.5 t$. The central peak disappears when the ferromagnetic spin correlations are eliminated at even larger negative $J$. More detail can be found in the text.

$\left.\left\langle{\overrightarrow{S_{\mathrm{D}}}}^{2}\right\rangle_{\text {cluster }}=S_{\mathrm{D}}\left(S_{\mathrm{D}}+1\right)\right)$, at $\Delta V=0.2$ and $V_{g}=-0.6$ (particle hole symmetry). Since the number of particles at this gate potential is 2, we deduce that the ground state can be expressed as a combination of states with $S_{\mathrm{D}}=1$ and 0 , with a weight of 86 percent for $S_{\mathrm{D}}=1$. In the case $\Delta V=t$, we obtain for the particle-hole symmetric gate potential a total spin $S_{\mathrm{D}}=0.352$, which corresponds to a 60 percent chance of having $S_{\mathrm{D}}=1$.

One way to confirm that the central peak in Fig. 4 a) indeed originates in a $S_{\mathrm{D}}=1$ state is by reducing the value of $J$, making less probable the ferromagnetic coupling between levels $\alpha$ and $\beta$. Figure 13 shows the conductance for the case shown in Fig. 4 a) plus two differents cases with the same values of $U, U^{\prime}$ and $t^{\prime}$ but different $J$ 's. In (b) with $J=0$ we observe a narrow central peak showing that the effect persists due to the $T_{\mathrm{K}(\mathrm{S}=1)}$ energy. For negative values of $J$, as shown in (c), the ferromagnetic state is even less probable and the central peak is more narrow. This peak must dissapear for larger negatives values of $J$ (i.e. $|J|>>T_{\mathrm{K}(\mathrm{S}=1)}$ ). All this evidence suggests that it is the spin-one Kondo effect that is responsible for some of the effects discussed here.

\section{UNDERSTANDING THE INTERFERENCE IN THE CONDUCTANCE}

The previous analysis showed that the origin of the three peaks in the conductance can be traced back to a Kondo effect that occurs for 1,2, and 3 electrons inside the dot. However, it remains to be understood what causes the conductance cancellation between them. It is expected that the two paths through $\alpha$ and $\beta$ - are responsible for the interference, but a more detailed explanation would be desirable. 


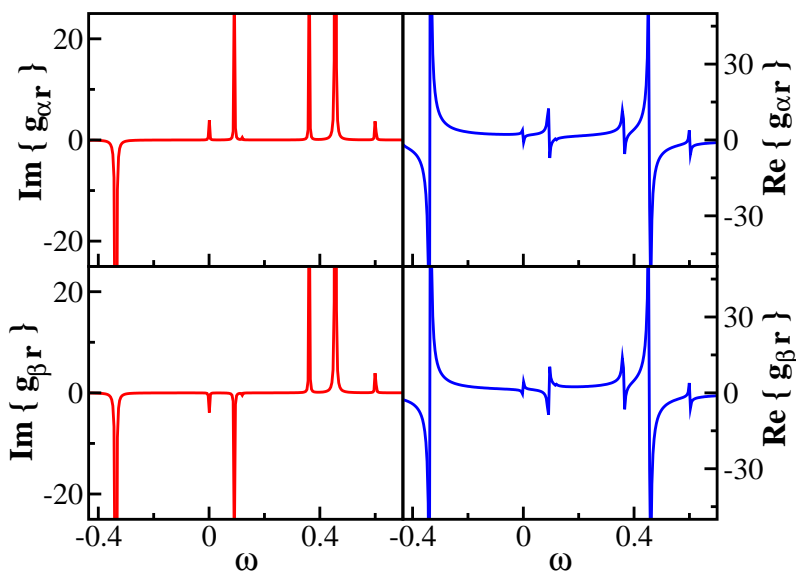

FIG. 14: Density-of-states of the bare $L=4$ cluster, for the cases $\Delta V=0.05 t$ and $V_{g}=-1.082 t$. The Fermi level lies at $\omega=0.0$. Note the relative change of the weight signs for the poles near $\omega=0$, when comparing the Green functions $g_{\alpha N}$ and $g_{\beta N}$.

\section{A. Origin of the $\pi$ Phase Difference}

To understand how the phase difference arises let us first study the cluster Green function. In Fig. 14 the real and the imaginary parts of $g_{\alpha r}$ and $g_{\beta r}$ are shown for the case of $\Delta V=0.05 t$ and a gate potential where the relative phase is $\pi$ $\left(V_{g}=0.108 t\right)$. Since the cluster is by definition a finite system, these two Green functions are simply a collection of a large number of poles. For $\Delta V=0$, level $\alpha$ is equal to $\beta$ in energy, and both Green functions must have poles at exactly the same positions. We expect that for a small $\Delta V$ their features must still remain similar. The most important information arising from Fig. 14 is that the poles at approximately the same energy corresponding to $g_{\alpha r}$ and $g_{\beta r}$ near the Fermi level $(\omega=0)$ have different signs in their weights. This means that those single poles have a phase difference of $\pi$, which causes the interference.

Other special property of the bare (cluster) Green function is shown in Fig. 15 In this figure, the imaginary parts of $g_{\alpha \alpha}$ and $g_{\alpha r}$ for $\Delta V=0.05 t$ and $\Delta V=0$ are shown. While for $\Delta V=0.05 t$ both Green functions have poles at the same positions, at $\Delta V=0$ the weight of the poles near the Fermi level for $g_{\alpha r}$ are zero. This is a characteristic of a localized state.

To understand these two properties we have to analyze the non-diagonal Green functions $g_{\alpha r}$ and $g_{\beta r}$ in their Lehmann representation ${ }^{24}$ :

$$
\begin{aligned}
g_{\alpha r}(\omega)= & \sum_{l} \frac{\left\langle f\left|c_{\alpha \sigma}\right| \phi_{l}\right\rangle\left\langle\phi_{l}\left|c_{r \sigma}^{+}\right| f\right\rangle}{\omega-\left(E_{\mathrm{F}}-E_{l}\right)+i \eta} \\
& +\sum_{l} \frac{\left\langle f\left|c_{r \sigma}^{+}\right| \phi_{l}\right\rangle\left\langle\phi_{l}\left|c_{\alpha \sigma}\right| f\right\rangle}{\omega+\left(E_{\mathrm{F}}-E_{l}\right)+i \eta} \\
g_{\beta r}(\omega)= & \sum_{l} \frac{\left\langle f\left|c_{\beta \sigma}\right| \phi_{l}\right\rangle\left\langle\phi_{l}\left|c_{r \sigma}^{+}\right| f\right\rangle}{\omega-\left(E_{\mathrm{F}}-E_{l}\right)+i \eta}
\end{aligned}
$$

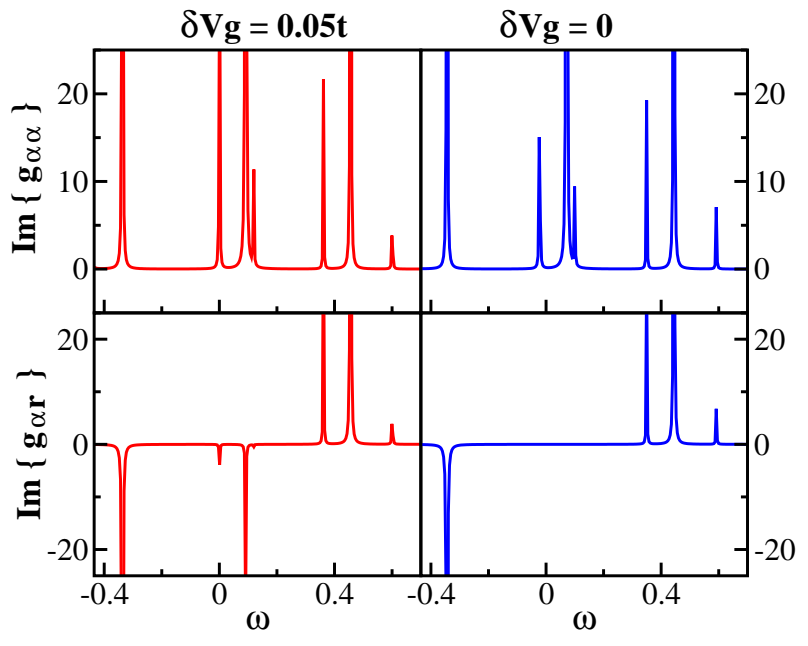

FIG. 15: Comparison between the imaginary parts of $g_{\alpha \alpha}$ and $g_{\alpha N}$, for the cases $\Delta V=0$ and $\Delta V=0.05 t$, working at $V_{g}=-1.082 t$ for the $L=4$ cluster. The pole in $g_{\alpha N}$ near the Fermi level disappears (i.e. carries no weight) at $\Delta V=0$.

$$
+\sum_{l} \frac{\left\langle f\left|c_{r \sigma}^{+}\right| \phi_{l}\right\rangle\left\langle\phi_{l}\left|c_{\beta \sigma}\right| f\right\rangle}{\omega+\left(E_{\mathrm{F}}-E_{l}\right)+i \eta}
$$

where $|f\rangle$ and $E_{\mathrm{F}}$ are the ground state and its eigenenergy, $\left\{\left|\phi_{l}\right\rangle\right\}$ is a complete basis of Hamiltonian eigenstates with eigenenergies $E_{l}$, and $\eta$ is an small number (typically $10^{-7}$ ).

Both Green functions have poles in the same positions $\omega= \pm\left(E_{\mathrm{F}}-E_{l}\right)$. For simplicity, only the first term in Eqs. 12 and 13 will be analyzed, but the study presented below is qualitatively the same for the second term. As the matrix element $\left\langle\phi_{l}\left|c_{r \sigma}^{+}\right| f\right\rangle$ is the same for both Green functions, it is clear that they only can have a phase difference in $\pi$ if the matrix element $\left\langle f\left|c_{\alpha \sigma}\right| \phi_{l}\right\rangle$ has a different sign than $\left\langle f\left|c_{\beta \sigma}\right| \phi_{l}\right\rangle$.

This change in the sign of the matrix elements can be understood based on the "reflection" symmetry between levels $\alpha$ and $\beta$. For the case $\Delta V=0$, the transformation $\alpha \rightarrow \beta$ must leave the Hamiltonian invariant. The operator $\hat{O}_{r}$ associated with this operation commutes with the total Hamiltonian and has eigenvalues 1 and -1 (if the states are even or odd under this transformation). The complete basis $\left\{\phi_{l}\right\}$ used in Eqs 12 and in 13 can be expanded in two new basis $\left\{\phi_{l}^{+}\right\}$and $\left\{\phi_{l}^{-}\right\}$ depending on whether the states are even or odd:

$$
\begin{aligned}
& \hat{O}_{r}\left|\phi_{l}^{+}\right\rangle=\left|\phi_{l}^{+}\right\rangle, \\
& \hat{O}_{r}\left|\phi_{l}^{-}\right\rangle=-\left|\phi_{l}^{-}\right\rangle .
\end{aligned}
$$

The destruction operators for the $\alpha$ and $\beta$ levels transform as

$$
c_{\alpha \sigma}=\hat{O}_{r}^{+} c_{\beta \sigma} \hat{O}_{r} .
$$

Suppose the ground state is an even state $\left|f^{+}\right\rangle$(we confirmed this numerically). Then, there are two kinds of matrix elements, $\left\langle f^{+}\left|c_{\alpha \sigma}\right| \phi_{l}^{+}\right\rangle$and $\left\langle f^{+}\left|c_{\alpha \sigma}\right| \phi_{l}^{-}\right\rangle$. These two matrix elements must be compared with their corresponding matrix elements, calculated with the operator $c_{\beta \sigma}$. Using Eqs 15 and 16. we have

$$
\left\langle f^{+}\left|c_{\alpha \sigma}\right| \phi_{l}^{+}\right\rangle=\left\langle f^{+}\left|c_{\beta \sigma}\right| \phi_{l}^{+}\right\rangle,
$$




$$
\left\langle f^{+}\left|c_{\alpha \sigma}\right| \phi_{l}^{-}\right\rangle=-\left\langle f^{+}\left|c_{\beta \sigma}\right| \phi_{l}^{-}\right\rangle .
$$

Then, some of the poles of Eqs 12 and 13 carry weight with differents signs. When $\Delta V$ is different from zero, the weights are different but the difference in the signs remains.

\section{B. Interference between $S=1 / 2$ and $S=1$ Kondo states?}

Note that the conductance cancellations mainly occur for values of the gate voltage where the charge of the dots is changing, for example from 1 to 2 . This suggests that an alternative way to visualize the interference process is to imagine the ground state in this intermediate regime as a linear combination of two Kondo states, one corresponding to a spin 1/2 at the dot and the other to a spin 1 at the same dot. In fact, in the important range of gate voltages considered in our study, the charge at the dot is not sharply defined, thus this approximation is reasonable. The interference could arise from a destructive process between the transport corresponding to each of these two nearly degenerate states. This hypothesis will be analyzed more extensively in future studies.

\section{Localized States}

Note that the matrix element $\left\langle f^{+}\left|c_{r \sigma}^{+}\right| \phi_{l}^{-}\right\rangle$must be zero when $\Delta V=0$, since the operator $c_{r \sigma}^{+}$does not change the symmetry of the state $\left|\phi_{l}^{-}\right\rangle$and the resulting state is orthogonal to $\left\langle f^{+}\right|$. This fact produces localized states in the QD due to the poles associated with the states $\left|\phi^{-}\right\rangle$(located at $\omega=E_{F}-E_{l}$ ) which are present in $g_{\alpha \alpha}$ and $g_{\beta \beta}$ (the element $\left\langle f^{+}\left|c_{r \sigma}^{+}\right| \phi_{l}^{-}\right\rangle$ does not appear in these Green functions), but they are not present in the Green function that transports electrons from one lead to the other $\left(g_{\alpha r}\right.$ or $\left.g_{l r}\right)$. This fact explains the results observed in the right frame of Fig. 15. When $\Delta V$ is not zero, this is no longer valid since $\hat{O}_{r}$ does not commute with $H_{\mathrm{T}}$.

One way to eliminate the symmetry between $\alpha$ and $\beta$ could be by using a different diagonal Coulomb repulsion for both levels. In Fig. 16 we can see the conductance, the phase difference, and the partial conductances for the case with $\Delta V=0$ and $U_{\beta}=U_{\alpha}+0.1 t$. Note that the first discontinuity remains since for one or zero electron in the QD, the different Coulomb repulsions $U_{\alpha}$ and $U_{\beta}$ are not active and the symmetry is not broken. In this case all the physics related to the poles associated with the symmetry $\alpha-\beta$ discussed above could be applied and the discontinuities shown in Fig. 22 a) appears.

\section{CONDUCTANCE CANCELLATION IN SIMILAR SYSTEMS}

In this section, we will discuss two other systems where a similar interference process is also operative.

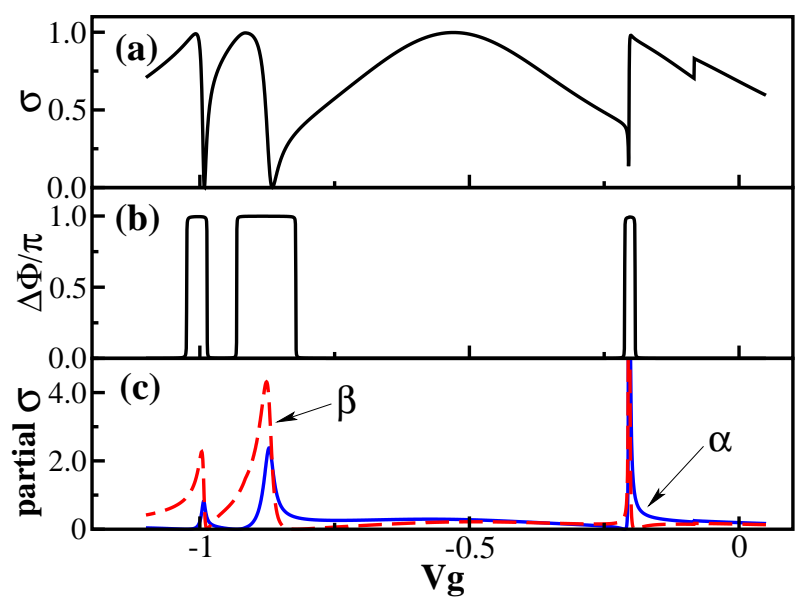

FIG. 16: Non-symmetric $\alpha-\beta$ case, where the Coulomb repulsions in both levels are different i.e. $U_{\beta}=U_{\alpha}+0.1 t$. Dips in the conductance are observed even with $\Delta V=0$, due to the explicitly broken symmetry. More details can be found in the text.

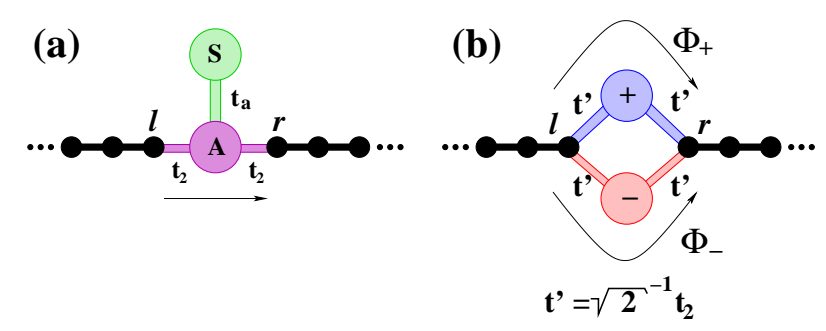

FIG. 17: (a) Schematic representation of a system of two coupled $\mathrm{QD}$, one of them side-connected. In this case, each dot has only one level. (b) Equivalent system after the transformation Eqs 19 and 20 The two possible paths in the new basis are shown.

\section{A. Two Coupled QD}

The first system to analyze consists of two coupled onelevel quantum dots, one of them side-connected ${ }^{25,26}$, as shown schematically in Fig. 177a). The two dots $(A$ and $S)$ are described by the Hamiltonian $H=H_{\text {dots }}+H_{\text {leads }}+H_{\text {int }}$, where

$$
\begin{aligned}
H_{\mathrm{dots}}= & \sum_{\sigma, \lambda=\mathrm{A}, \mathrm{S}}\left\{U / 2 n_{\lambda \sigma} n_{\lambda \bar{\sigma}}+V_{g} n_{\lambda \sigma}\right\}+ \\
& t_{a} \sum_{\sigma} c_{\mathrm{A} \sigma}^{+} c_{\mathrm{S} \sigma}+\text { h.c. } \\
H_{\mathrm{int}}= & t_{2} \sum_{\sigma} c_{\mathrm{A} \sigma}^{+}\left[c_{l \sigma}+c_{r \sigma}\right]+\text { h.c. }
\end{aligned}
$$

As these are two physically separated quantum dots, we will neglect the terms $U^{\prime}$ and $J$ used in the previous section. Here, $\operatorname{dot} A$ is the only one connected to the leads, by a matrix element $t_{2} . H_{\text {lead }}$ is the same as in previous sections.

Considering the two dots as a "dimer", $H_{\text {dots }}$ can be rewrit- 


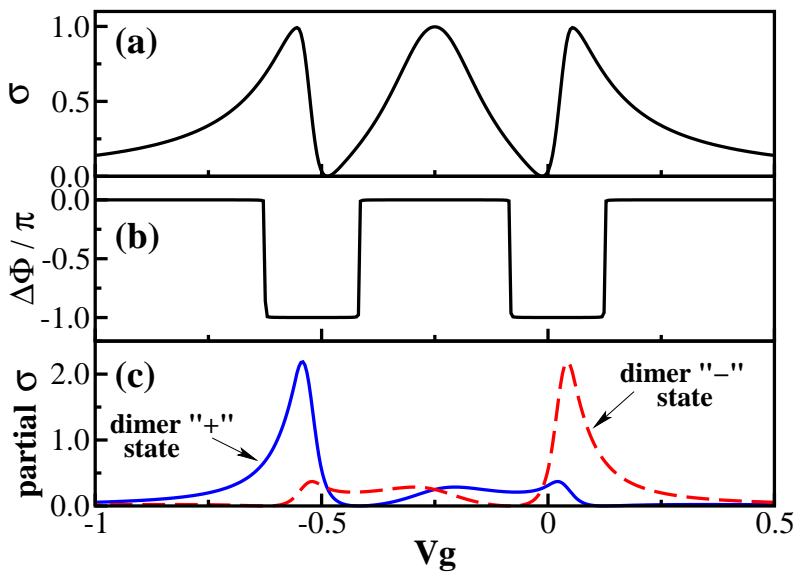

FIG. 18: Conductance (in units of $e^{2} / h$ ), phase difference and partial conductances as a function of the gate potential $V_{g}$, for the geometry shown in Fig 17 The parameters used, in units of $t$ (the bandwidth is $W=4 t$ ), are $t_{a}=0.12, t_{2}=0.25$ and $U_{i}=0.5$. In (a), two zeros in the conductance as a function of $V_{g}$ are shown. (b) The phase difference $\Delta \Phi$ near these zeros jumps to $-\pi$. In (c), the partials contributions to the conductance $\sigma_{-}$and $\sigma_{+}$are given. For $V_{g}=-U_{i} / 2$, we can observe a constructive interference process where $\sigma_{-}=\sigma_{+}$and $\Delta \Phi=0$.

ten in the basis of bonding and anti-bonding $\operatorname{states}^{27}$,

$$
\begin{aligned}
& c_{+\sigma}=\frac{1}{\sqrt{2}}\left[c_{\mathrm{A} \sigma}+c_{\mathrm{S} \sigma}\right], \\
& c_{-\sigma}=\frac{1}{\sqrt{2}}\left[c_{\mathrm{A} \sigma}-c_{\mathrm{S} \sigma}\right] .
\end{aligned}
$$

With this transformation, it is easy to visualize the two possible paths that produce the interference in the conductance (shown in Fig. 17). In the new basis, the matrix element that connects the dimer state with the leads is $t^{\prime}=1 / \sqrt{2} t_{2}$. Note that the total charge in the two dots does not depend on the basis used, since $\left\langle n_{\mathrm{A} \sigma}+n_{\mathrm{s} \sigma}\right\rangle=\left\langle n_{-\sigma}+n_{+\sigma}\right\rangle$. Also note that this bonding and anti-bonding states have a diagonal single energy of value $V_{g-}=V_{g}-t_{a}$ and $V_{g+}=V_{g}+t_{a}$. For this reason, the matrix element $t_{a}$ plays the same role that $2 \Delta V$ had in the case of a quantum dot with two levels analyzed before.

As in the previous sections, we can define the conductance as a function of the partial conductances through channels "+" and "-", as well as the phase difference between them as $\sigma=\sigma_{+}+\sigma_{-}+\sqrt{\sigma_{+} \sigma_{-}} \cos (\Delta \Phi)$. In Fig. 18, we show the results for the conductance, phase and partial conductances $\sigma_{+}$and $\sigma_{-}$for the case equivalent to that presented in Fig. 2 b. Here, the dot coupling factor $t_{a}$ used is $0.12 t$. Again, a threepeaks structure in the conductance can be observed. The zeros in the conductance appear when the phase jumps to $-\pi$. This is a destructive interference process similar to that shown in Fig. 4 for $\Delta V=0.2 t$.

As in section III the results presented here correspond to the smallest cluster size $L=4$. In the final part of this subsection the convergence with the size $L$ will be analyzed. In Fig. 19 the conductance and the charge of the dimer states and dots are shown. The charge of the dimer states presents the

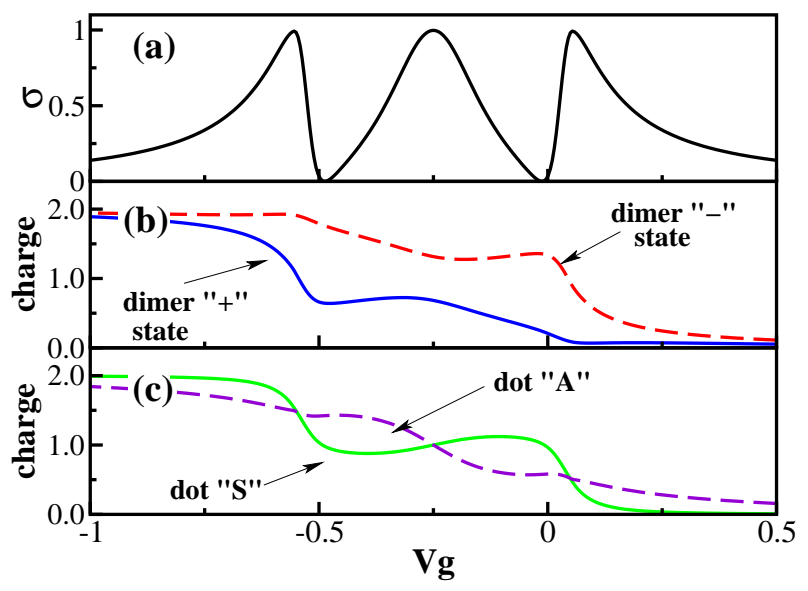

FIG. 19: Conductance (in units of $e^{2} / h$ ) and charge as a function of $V_{g}$, for the same parameters used in Fig. 18 Shown are (a) the conductance vs. $V_{g}$; (b) the charge at the bonding and anti-bonding dimer states; (c) the charge at the real dots "A" and "S".

same behavior as the charge of the two-level dot studied before. The charge in the individual dots shows a slow increase for dot "A", and a step-function-like behaviour for dot " $\mathrm{S}$ ". This indicates that while the dot coupled to the leads is in a transition-valence regime and the charge enters slowly due to the Kondo resonance, the other one is in a Coulomb-blockade regime.

Increasing $t_{a}$ the dimer states are splitted and the two-dots system can now be represented by one QD with two levels, with a large $\Delta V \sim 2 t_{a}$. This is shown in Fig. 20 In the last two figures (Figs. 21 22 the convergence with the cluster size is shown for the conductance and the charge. As before, a fast convergence is observed. In the case of the charge of dot "S", note that the wavy behavior found for the smaller cluster is replaced by a "plateaux" when the cluster-size increased.

\section{B. Two coupled QD with two levels}

In this subsection, it will be analyzed the case of two QD in series, each with two levels, as schematically shown in Fig. 23. The Hamiltonian that describes this problem includes

$$
\begin{aligned}
H_{\mathrm{dots}}= & \sum_{i=1,2} \sum_{\sigma, \lambda=\alpha \beta} U / 2 n_{i \lambda \sigma} n_{i \lambda \bar{\sigma}}+ \\
& U^{\prime} / 2 \sum_{\sigma \sigma^{\prime}} n_{i \alpha \sigma} n_{i \beta \sigma^{\prime}}+ \\
& -J \sum_{\sigma \sigma^{\prime}} c_{i \alpha \sigma}^{+} c_{i \alpha \sigma^{\prime}} c_{i \beta \sigma^{\prime}}^{+} c_{i \beta \sigma}+ \\
& \left.V_{g} \sum_{\sigma}\left[n_{i \alpha \sigma}+\left(V_{g}+\Delta V\right) n_{i \beta \sigma}\right]\right\}+ \\
& \sum_{\sigma, \lambda=\alpha \beta} t^{\prime \prime}\left[c_{1 \lambda \sigma}^{+} c_{2 \lambda \sigma}+\text { c.c. }\right]
\end{aligned}
$$




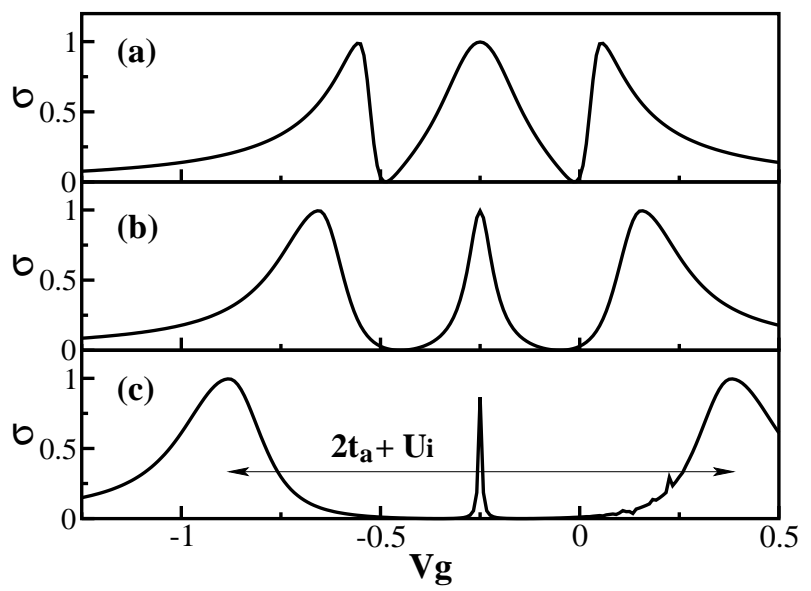

FIG. 20: Conductance (in units of $e^{2} / h$ ) for three different values of $t_{a}$. (a) $t_{a}=0.12$ (reproduced here for comparison), (b) $t_{a}=0.24$, and (c) $t_{a}=0.48$. For $t_{a}=0$ the side coupled dot is not connected and the conductance presents the characteristics of one QD (not shown). Increasing $t_{a}$, the side coupled dot starts to participate in the transport, and interference effects appear. For large $t_{a}$, the system - represented by the dimer states - is equivalent to one dot with two levels, separated by an energy $2 t_{a}+U$.

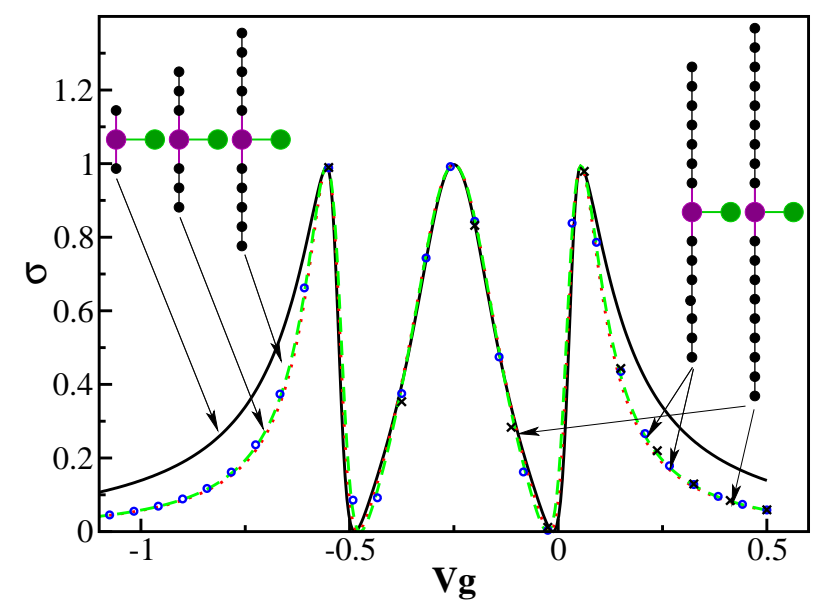

FIG. 21: Convergence of the conductance with the cluster size, for the case of the side-connected dot. The parameters are the same as in Fig. 18

$$
H_{\mathrm{int}}=t^{\prime} \sum_{\sigma, \lambda=\alpha, \beta}\left[c_{1 \lambda \sigma}^{+} c_{l 0 \sigma}+c_{2 \lambda \sigma}^{+} c_{r 0 \sigma}+\text { c.c. }\right]
$$

where $c_{i \lambda \sigma}^{+}$creates an electron at the dot $i$ in the level $\lambda$ with spin $\sigma$. The Hamiltonian which describes the leads is the same as before. Then, the total Hamiltonian is $H_{\mathrm{T}}=H_{\mathrm{dot}}+H_{\text {leads }}+$ $H_{\text {int }}$. As in the case of two QD with one level each, we can rewrite this problem in the dimer-state basis. It is well known that in this kind of systems, a splitting in energy between the dimer states must occur $21,28,29.30$. This separation is related to the $t^{\prime \prime}$ matrix element.

As before we present the results corresponding to the smallest cluster size. For this case $L=6$ that corresponds to two sites to describe the "leads" and four levels to describe the

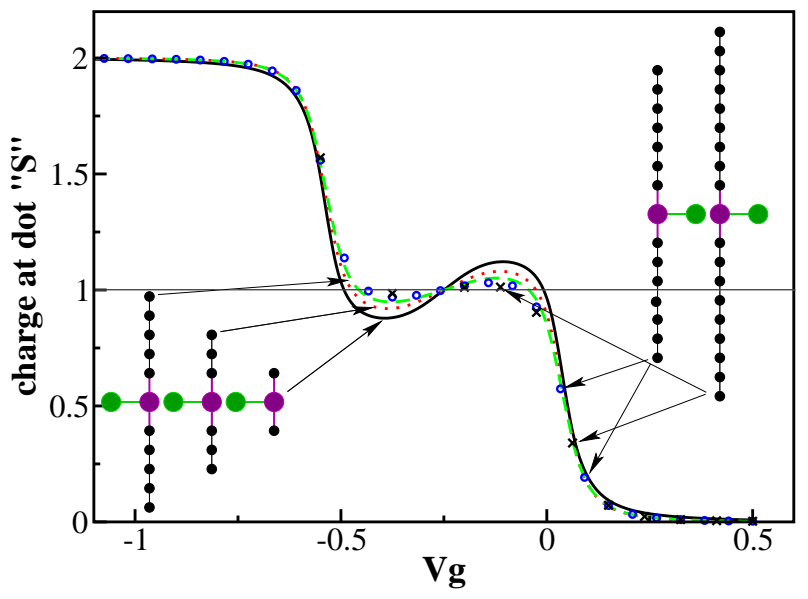

FIG. 22: Convergence study of the charge at dot "S" increasing the size of the cluster exactly solved. For the smaller cluster, we found important changes around $V_{g}=-U / 2$, but eventually the system converges to a plateaux for a sufficiently large cluster. The parameters are the same as in Fig. 18

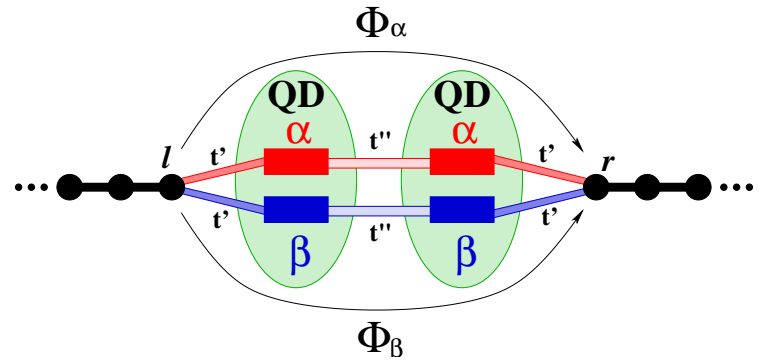

FIG. 23: Schematic representation of the system of two coupled QD with two levels. The two possible paths carrying different phases $\Phi_{\alpha}$ and $\Phi_{\beta}$ are shown.

two dots with two levels each. Figure 24 is the equivalent of Fig 2 for the case of only one QD. The conductance for several $\Delta V$ 's is shown. As in the previous case, at large $\Delta V$ there are two splitted structures corresponding to the Kondo regime for the $2 \mathrm{QD}$ with one level. An intermediate case $\Delta V=0.9 t$, where these two structures start to merge, is also shown. When $\Delta V=0$, similar structures can be observed as found in previous cases, where every discontinuity was splitted in two due to the dimer bonding anti-bonding states.

In Figures 25 and 26 the conductance and the phase for a very small $\Delta V=0.01 t$ are presented. In this case the $\Delta V=0$ discontinuities are transformed into narrow dips. In Fig. 26 the first two dips are shown. They qualitatively correspond to the splitting of the first dip in the 1QD case. The phase in Fig 26 does not present the abrupt jumps that were found for 1QD, but the dips nevertheless occur when the phase takes the value $\Delta \Phi= \pm \pi$. 


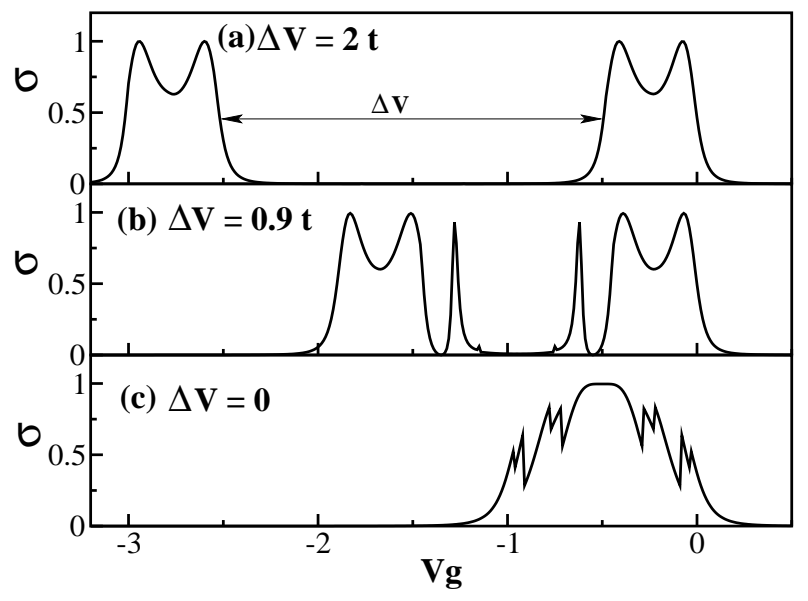

FIG. 24: Conductance of two coupled quantum dots, each with two levels, for three different cases of $\Delta V$ (large, intermediate, and zero). (a) When $\Delta V$ is the largest energy, two structures separated by $\Delta V$ appear in the conductance, similarly as for two coupled one-level QDs. (b) When $\Delta V$ decreases these two structures start to overlap producing dips in the conductance. (c) A more complex conductance structure with several discontinuities is obtained in the limit $\Delta V=0$.

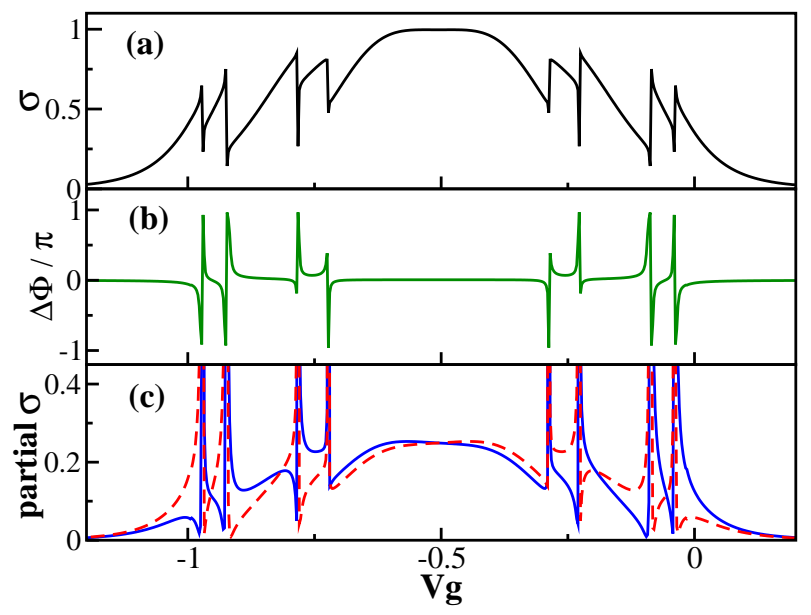

FIG. 25: Conductance (in units of $e^{2} / h$ ), phase, and partial conductances for a small $\Delta V=0.01 t$, in the case of two coupled twolevels QDs. Typical dips in the conductance will be analyzed in the next figure. Near the gate potential $V_{g}=-0.5$, a constructive interference occurs. The phase is zero and the partial conductance becomes $\sim 0.25$, inducing a constructive phase process for electron transport.

\section{CONCLUSIONS}

In this paper, we have investigated systems where interference effects in the conductance can arise in multilevel quantum dots. Although there are no obvious different paths, as in a $\mathrm{AB}$ interferometer, transport using different levels inside the QD can produce such interference. Using an exact diagonalization plus embedding technique the phase difference between these paths was calculated. When this phase difference is $\pm \pi$, a destructive interference can appear. An important aspect of these results is that the conductance cancellations

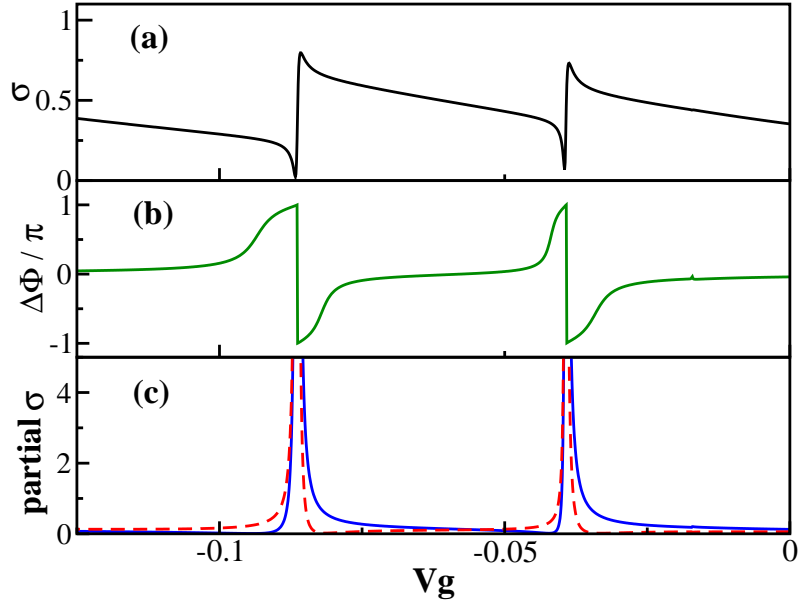

FIG. 26: First two dips in the conductance for the small $\Delta V$ case shown in Fig. 25 Both partial conductances $\left(\sigma_{\alpha}\right.$ and $\left.\sigma_{\beta}\right)$ have a zero near the dips, but not at the same time. Then, the dip in the overall conductance is produced by a phase interference process induced by $\Delta \Phi= \pm \pi$.

can occur in the absence of external magnetic fields - usually used in $\mathrm{AB}$ experiments - and also without introducing at the Hamiltonian level any relative phase for the hopping amplitudes of the states involved. The Coulombic interaction is the main responsible for the effect, which can also be visualized as an interference between $S_{\mathrm{D}}=1$ and $1 / 2$ Kondo states.

Another important novel effect found in this investigation is the possibility of having localized states inside the QDs. These states do not contribute to the conductance but they modify the many-body physics of the systems through Coulombic effects. These localized states induce discontinuities in the conductance vs. gate voltage for particular couplings, while in the most general case conductance cancellations are observed.

The results presented in this paper will hopefully provide motivation to experimentalists to refine their measurements to search for conductance cancellations. While our conductance vs. gate voltage curves should be cautiously used to compare against experiments - since the details of the results vary as a function of parameters, and in addition most results were obtained using only two active levels - the ideas introduced here are expected to be robust. When multilevel quantum dots with interacting electrons are considered, cancellations are the rule more than the exception.

Experimental realizations of quantum dots with many active levels are possible. Recently, Aikawa et al ${ }^{31}$ observed that quantum-dot states can be classified into a small number of states that are strongly coupled to the leads, and a large number of states weakly coupled to those leads. The mixing between these states was found to be of relevance to explain transport experiments. It is clear that many theoretical calculations must be revisited to incorporate the multiplicity of levels in dots. This is also important if transport properties 
through atoms are studied. This is more challenging experimentally than the study of relatively larger size quantum dots, but possible. In the case of atoms, degeneracies are natural in open-shell atoms, and a variety of interference processes as those observed here are possible.

\section{ACKNOWLEDGMENTS}

The authors acknowledge conversations with E.V. Anda, M.A. Davidovich, G. Chiappe and M.V. Apel. Support was provided by the NSF grants DMR-0122523 and 0303348. Additional funds have been provided by Martech (FSU).
${ }^{1}$ E. Dagotto, Nanoscale Phase Separation and Colossal Magnetoresistance, Springer-Verlag, Berlin, 2002; Colossal Magnetoresistance Oxides, edited by Y. Tokura, Gordon \& Breach, New York, 2000. A. Moreo, S. Yunoki and E. Dagotto, Science 283, 2034 (1999); Y. Tokura and N. Nagaosa, Science 288, 462 (2000); M. Uehara, S. Mori, C. H. Chen, and S.-W. Cheong, Nature 399, 560 (1999); J. W. Lynn, R. W. Erwin, J. A. Borchers, Q. Huang, A. Santoro, J-L. Peng and Z. Y. Li, Phys. Rev. Lett. 76, 4046 (1996), and J. M. De Teresa, M. R. Ibarra, P. A. Algarabel, C. Ritter, C. Marquina, J. Blasco, J. García, A. del Moral, Z. Arnold, Nature 386, 256 (1997); P. G. Radaelli, R. M. Ibberson, D. N. Argyriou, H. Casalta, K. H. Andersen, S.-W. Cheong, and J. F. Mitchell, Phys. Rev. B 63, 172419 (2001); D. N. Argyriou, J. W. Lynn, R. Osborn, B. Campbell, J. F. Mitchell, U. Ruett, H. N. Bordallo, A. Wildes, and C. D. Ling, Phys. Rev. Lett. 89, 036401 (2002). In the context of cuprates see: S. H. Pan, J. P. O'Neal, R. L. Badzey, C. Chamon, H. Ding, J. R. Engelbrecht, Z. Wang, H. Eisaki, S. Uchida, A. K. Gupta, K.-W. Ng, E. W. Hudson, K. M. Lang, J. C. Davis, Nature 413, 282 (2001). See also J. M. Tranquada, B. J. Sternlieb, J. D. Axe, Y. Nakamura, S. Uchida, Nature 375, 561 (1995).

2 A.C. Hewson, The Kondo Problem to Heavy Fermions (Cambridge University Press, Cambridge, UK, 1993)

3 L.I. Glazman and M.E. Raikh, Pis'ma Zh Éksp. Teor. Fiz.47, 378 (1988) [JETP Lett. 47, 452 (1988)]. T.K. Ng and P.A. Lee, Phys. Rev. Lett 61, 1768 (1988). Y. Meir and N.S. Wingreen, Phys. Rev. Lett. 68, 2512 (1992).

4 D. Goldhaber-Gordon, H. Shtrikman, D. Mahalu, David AbuschMagder, U. Meirav, A. Kastner, Nature 391, 156 (1998); S.M. Cronenwett, T. H. Oosterkamp, and L. P. Kouwenhoven, Science 281, 540 (1998).

5 D. Loss and E.V. Sukhorukov, Phys. Rev. Lett. 84, 1035 (2000).

${ }^{6}$ R. López, D. Sanchez, M. Lee, M-S. Choi, P. Simon and K. Le Hur, cond-mat/0402361 R. López, R. Aguado, and G. Platero, Phys. Rev. Lett. 89, 136802 (2002). R. Aguado and D.C. Langreth, Phys. Rev. Lett. 85, 1946(2000).

7 D. Boese, W. Hofstetter and H. Schoeller, Phys. Rev. B 64, 125309 (2001).

${ }^{8}$ M. L. Ladrón de Guevara, F. Claro, and P. A. Orellana, Phys. Rev. B 67, 195335 (2003). See also Z. Y. Zeng, F. Claro, and A. Pérez, Phys. Rev. B, 085308 (2002).

9 T-S. Kim and S. Hershfield, Phys. Rev. B67 235330 (2003). See also A. Silva, Y. Oreg, and Y. Gefen, cond-mat/0205436

10 C.A. Büsser, Adriana Moreo and Elbio Dagotto, submitted to PRB, cond-mat/0308413 See also G. Chiappe and J. A. Vergés, cond-mat/0309458

11 S. Sasaki, S. De Franceschi, J.M. Elzernman, W.G. van der Wier,
M. Eto, S. Tarucha and L.P. Kouwenhoven, Nature 405, 764 (2000).

12 M. Pustilnik and L.I. Glazman, Phys. Rev. Lett. 85, 2993 (2000).

13 M.A. Davidovich, E.V. Anda, C.A. Büsser and G. Chiappe. Phys. Rev. B 65, 233310 (2002).

14 A. Yacoby, M. Heiblum, D. Mahalu, and Hadas Shtrikman, Phys. Rev. Lett. 74, 4047 (1995).

15 W. Hofstetter and H. Schoeller. Phys. Rev. Lett. 88, 016803 (2002). See also D. Boese, W. Hofstetter and H. Schoeller, Phys. Rev. B 66, 125315 (2002). Interference studies also without using magnetic fields or different pathways but based on Fano resonances can be found in S-J. Xiong and Yue Yin, Phys. Rev. B 66 153315 (2002).

16 Y. Meir, N.S. Wingreen and P.A. Lee, Phys. Rev. Lett. 66, 3048 (1991).

17 J.A. Appelbaum and D.R. Penn, Phys. Rev. 188, 874 (1969). C. Lacroix, J. Phys. F 11, 2389 (1981). Y. Meir, N.S. Wingreen and P.A. Lee, Phys. Rev. Lett. 70, 2601 (1993).

18 Note that the equation of motion for $G_{l r}$ take this simple form due the sites $l$ and $r$ have no many-body interactions.

19 E. Dagotto, Rev. Mod. Phys 66, 763 (1994).

20 V. Ferrari, G. Chiappe, E.V. Anda, M.A. Davidovich, Phys Rev Lett 82, 5088, (1999).

${ }^{21}$ C.A. Büsser, E.V. Anda, A.L. Lima, Maria A. Davidovich and G. Chiappe, Phys. Rev. B 62, 9907 (2000).

22 E. Dagotto, T. Hotta, and A. Moreo, Physics Reports 344, 1 (2001). See also J. Kuei and R. T. Scalettar, Phys. Rev. B 55, 14968 (1997); A.L. Malvezzi and E. Dagotto, Phys. Rev. B 63, 140409 (2001).

${ }^{23}$ D. Golhaber-Gordon, J. Göres, Hadas Shtrikman, D. Mahalu, U. Meirav, M.A. Kastner, contribution to $24^{\text {th }}$ International Conference on the Physics of Semiconductors, D. Gershoni ed., Jerusalem, Israel (1998).

24 J.W. Negele and H. Orland, Quantum Many-Partice Systems, (Advanced Book Classics, 1998).

25 V.M. Apel, M.A. Davidovich, E.V. Anda, C.A. Büsser and G. Chaippe, Microelectronics J. 34, 729 (2003).

26 T-S. Kim and S. Hershfield, Phys. Rev. B63, 245326 (2001).

27 V.M. Apel, M.A. Davidovich and E.V. Anda, preprint (submitted to Phys. Rev. B).

${ }^{28}$ W. G. van der Wiel, S. De Franceschi, J. M. Elzerman, T. Fujisawa, S. Tarucha, and L. P. Kouwenhoven, Rev. Mod. Phys. 75, 1 (2003).

29 T. Aono, Eto and W. Kawamura, J. Phys. Soc. Jpn. 67, 1860 (1998). A. Georges, Y. Meir. Phys. Rev. Lett. 82, 3508, (1999).

${ }^{30}$ H. Jeong, A.M. Chang, M.R. Melloch, Science 2932221 (2001).

31 H. Aikawa, K. Kobayashi, A. Sano, S. Katsumoto, and Y. Iye, 
cond-mat/0312431 